\title{
Nurse led transitional care improved health related quality of life and reduced emergency department use for heart failure
}

\author{
Harrison MB, Browne GB, Roberts J, et al. Quality of life of individuals with heart failure: a randomised trial of the \\ effectiveness of two models of hospital-to-home transition. Med Care 2002;40:271-82.

\section{QUESTION: In patients admitted to hospital with heart failure, is nurse led transitional care more effective than usual care for improving health related quality of life and reducing use of health services?}

\section{Design}

Randomised (allocation concealed), blinded \{data collectors and data analysts\},* controlled trial with follow up 12 weeks after hospital discharge.

\section{Setting}

A large urban teaching hospital in Ottawa, Ontario, Canada.

\section{Patients}

192 patients (mean age 76 y, 55\% men) who were admitted to a general medical unit with congestive heart failure were included if they resided in the regional home care radius, expected to be discharged with home nursing care, were admitted for $>24$ hours to the nursing units, and were not cognitively impaired. Follow up was $82 \%$.

\section{Intervention}

Patients were allocated to a nurse led transitional care intervention $(\mathrm{n}=92)$ or usual care $(\mathrm{n}=100)$. The nurse led transitional care intervention was implemented from admission to 2 weeks after hospital discharge and included usual care plus a comprehensive programme for improving hospital to home transfer. The programme provided patient education, supportive care for self management, and linkages among healthcare providers and patients (eg, nursing transfer letter and telephone outreach). Usual care included usual discharge planning and post-discharge care by a multidisciplinary team; the timing and number of nurse home visits for this group matched those received by the transitional care group.

\section{Main outcome measures}

Health related quality of life, symptom distress, and function were measured by the Minnesota Living with Heart Failure Questionnaire (MLHFQ) and the Medical Outcome Study Short Form Questionnaire (SF-36). Secondary outcomes were all cause hospital readmissions and emergency department (ED) visits.

\section{Main results}

Analysis was by intention to treat. Disease specific health related quality of life improved more for patients in the transitional care group than for those in the usual care group, as shown by the mean MLHFQ total scores $(\mathrm{p} \leq 0.002)$ and mean MLHFQ physical dimension scores $(\mathrm{p} \leq 0.01)$ at 6 and 12 weeks, and by the mean MLHFQ emotional dimension scores at 6 weeks $(\mathrm{p}=0.006)$. The groups did not differ for quality of life measured by the SF-36 general health, physical component, or mental component scales. Patients in the transitional care group had fewer first ED visits and multiple ED visits than those in the usual care group (table); no difference existed between groups for hospital readmissions.

\section{Conclusion}

In patients with heart failure, a nurse led transitional care intervention improved health related quality of life and reduced use of the emergency department.

*Information provided by author.

Sources of funding:

Health Canada,

National Health

Research and

Development Program.

For correspondence: Dr M B Harrison,

Oueen's University,

Kingstom, Ontario,

Canada.

harrisnm@

post.queensu.ca

Transitional care $(T C)$ v usual care (UC) for emergency department $(E D)$ visits in patients with heart failure at 12 weekst

\begin{tabular}{lllll} 
Outcomes & TC & UC & RRR (95\% CI) & NNT (CI) \\
\hline First ED visit & $29 \%$ & $46 \%$ & $38 \%(6$ to 59$)$ & $6(3$ to 48$)$ \\
\hline$>1$ ED visit & $32 \%$ & $50 \%$ & $35 \%(5$ to 56$)$ & 6 (3 to 50$)$ \\
\hline
\end{tabular}

†Abbreviations defined in glossary; RRR, NNT, and $\mathrm{Cl}$ calculated from data in article.

\section{COMMENTARY}

The study by Harrison $e t$ al is timely and contributes to the evidence that nurse led interventions for patients with heart failure can improve quality of life and reduce use of health services. A unique feature of this study is that it focused on enhancing the transition from hospital to home by slightly altering the practices of existing staff, rather than by replacing the usual care nurse with a specialist nurse.

Quality of life was measured using the generic SF-36 and the disease specific MLHFQ. Disease specific quality of life was improved in patients who received enhanced transitional care. No changes in generic quality of life were observed. This finding adds to accumulating evidence that quality of life measures need to be carefully selected in research that examines the effect of interventions for patients with specific conditions.

The patients in this study were representative of the population with heart failure in terms of age and comorbidity. All patients had home care coordinator services and received $\geq 2$ home visits from community nurses within the first 2 weeks after discharge. Direct contact was made between hospital and home care nurses for all patients. This level of discharge care and communication for all participants was essential to the design, providing the standard against which the intervention could be appropriately tested. However, in many settings where such optimal discharge and communication processes do not exist, they alone could potentially improve patient outcomes. This may be an area for future research.

This study shows that small reorganisations and improvements in patient care during the transition from hospital to community can lead to short term benefits and can be done with existing staff. Whether improvements in quality of life beyond 3 months after discharge can be achieved still needs to be investigated to determine the sustainability of this intervention.

Nicola Williams, RGN, MScN Cardiology Nurse Specialist North Middlesex Hospital NHS Trust, London, UK 